\title{
Output-Feedback MRAC with Reference Model Tolerance of Nonlinearly Perturbed Delayed Plants
}

\author{
Boris Mirkin * Per-Olof Gutman* J. Sjöberg ** \\ * Technion-Israel Institute of Technology, Haifa 32000, Israel (e-mail: \\ bmirkin@technion.ac.il; peo@technion.ac.il). \\ ** Chalmers University of Technology, Department of Signals \& Systems, \\ SE41296 Gothenburg, Sweden (e-mail: Jonas.Sjoberg@chalmers.se)
}

\begin{abstract}
A novel approach within the conventional MRAC framework for solving the problem of robust adaptive output tracking for a class of uncertain nonlinear dynamical systems with unknown timevarying state delays is proposed. A MRAC problem formulation in addition to the traditional asymptotic zero error tracking specification makes the problem statement contain an additional explicit requirement, namely the tracking objective is described by a set of admissible reference trajectories which is called a performance tube. As the design parameter which specifies the size of the performance tube, we select the reference model input signal than can be changed within specified bounds. An additional optimization task is formulated to find the best trajectory with a cost function that penalizes the deviation of the control signal from a given signal.
\end{abstract}

Keywords: Direct adaptive control; Nonlinear Time-delay systems; Reference model tolerance.

\section{INTRODUCTION}

Model reference adaptive control (MRAC) is one of the main approaches to adaptive control, see e.g. the popular textbooks Narendra and Annaswamy (1989); Åström and Wittenmark (1995); Krstić et al. (1995); Ioannou and Sun (1996); Tao (2003); Ioannou and Fidan (2006). Most model reference adaptive control design techniques have only paid attention to control problem solutions for one particular performance index the tracking error which is the difference between the plant output and the reference model output. The reference model is chosen to generate a single desired trajectory that the plant output has to follow.

In many applications, however, it may be permissible to have an admissible set of reference trajectories which may lead to a possibly significant improvement of performance. Such a definition of reference trajectoires appears in flight control design, in the control of space structures and in industrial process control. While this reasoning makes sence, to date the real stumbling-block to its practical use is the lack of tractable algorithmic methods to guarantee for performance. The problem of how to determine a formal mechanism for the selection of an admissible set of reference trajectories is obviously important. This paper aims to open new directions to address this problem. It is shown that the desired result can be achieved by forming an additional optimization task and solve it. The optimality criterion penalizes the deviation of the control signal from a given signal.

In Mirkin and Mirkin (1999) we used an interval reference model to reduce the cost of control. The controller selects online a suitable reference trajectory from an a priory known admissible set. To find the best trajectory is formulated as an additional optimization task. The main idea of this method

* This work was supported by the Israel Science Foundation under Grant $588 / 07$. follows from the fact that if the input matrix $B$ of a linear plant with parameter uncertainties in the usual state-feedback MRAC formulation is known and equal the input matrix $B_{r}$ of a reference model, then the basic tracking error equation which is used for stability analysis and the adaptation algorithms design does not depend explicitly on the command signal of the reference model, see e.g. (Ioannou and Sun, 1996, p.325) . Therefore it is possible to consider the command input of the reference model as a design parameter and to change it within allowed limits. Its variability does not change neither the stability properties nor the synthesis of the adaptation algorithms. And these limits in turn determine the set of admissible reference trajectories.

But the result in Mirkin and Mirkin (1999) is applicable only to state-feedback control of linear systems with parametric uncertainty and without disturbances. Unfortunately it requires an exact knowledge of the input matrix which moreover has to equal to the reference model input matrix. It turns out to be quite difficult overcome that restriction. That research direction requires effective output-feedback designs to handle enlarged classes of nonlinear systems with disturbances, and time delays.

Therefore, in the current work, we focus on the problem of output-feedback MRAC when the tracking objective is described by an admissible set of reference trajectories called a performance tube, by which we propose a way to overcome the above mentioned difficulties. We consider a class of dynamic systems with unknown parameters and subject to unknown time-varying delays, nonlinear perturbations and external disturbances with unknown bounds.

\section{DESCRIPTION OF THE APPROACH}

The proposed design approach is based on the concept of goal adaptation as a way to ensure additional desirable properties of the closed-loop controlled systems. Goal adaptation technique as a way to formalize additional design requirements was intro- 
duced in our previous papers, see e.g. the review paper Mirkin (2001). In the framework of this concept, we now propose a new paradigm of performance shaping in the traditional MRAC setting.

The approach is as follows: Let us assume that it is possible to change, within prescribed bounds, a part of the reference signal $r$ to the reference model, or some of the parameters of its operator $W_{r}$. Let us call these the tolerance control $u_{c}$. Hence, tuning $u_{c}$ in prescribed bounds we form a admissible set of the reference trajectories which we will call - a performance tube. The performance tube has the property that each of its reference trajectory satisfies the specification. The adaptive control algorithm chooses, on-line, a desired trajectory from the performance tube based on measurements of the error $e(t)$ by varying the tolerance control component $u_{c}$. The main problems here are to determine a formal mechanism how to choose a reference trajectory from the performance tube, and how to find $u_{c}$ such that the resulting problem is tractable.

To achieve the stated aims we formulate our goal in the form of two control objectives - primary and secondary: (i) to achieve that the plant output asymptotically exact follows the output of any reference trajectory from a performance tube; (ii) to receive some additional benefit by varying the tolerance control $u_{c}(t)$ within specified limits.

The proposed design procedure has two phases. In the first phase, to achieve ( $i$ ) we find a control law parametrization in order to ensure asymptotical exact model tracking for any admissible $u_{c}(t)$. In the second phase, to get (ii), an optimization problem is formulated and solved.

\section{FORMAL PROBLEM STATEMENT}

\subsection{Model}

Let the uncertain single-input, $u(t)$, single-output, $y(t)$ plant with time delays appropriately initialized be given by

$$
\begin{aligned}
\dot{x}(t)= & A x(t)+A_{\tau} x\left(t-\tau_{x}(t)\right)+b u(t)+b f\left(y(t), y\left(t-\tau_{y}(t)\right), t\right) \\
& +b d(t) \\
y(t)= & c^{T} x(t)
\end{aligned}
$$

where $x(t) \in \mathbb{R}^{n}, y(t), u(t) \in \mathbb{R}, f\left(y(t), y\left(t-\tau_{y}(t)\right), t\right) \in \mathbb{R}$ and $d(t) \in \mathbb{R}$ are, respectively, the state, output, control input, nonlinear delayed perturbation and external disturbance. The constant matrices $A, A_{\tau}$ and the vectors $b$ and $c$ of appropriate dimensions have unknown elements. The time-delays $\tau_{x}(t)$ and $\tau_{y}(t)$ are nonnegative differentiable functions, satisfying

$$
\begin{aligned}
& 0 \leq \tau_{x}(t) \leq \tau_{x \max }, \\
& 0 \leq \tau_{y}(t) \leq \tau_{y \max }, \quad \dot{\tau}_{y}(t) \leq \tau_{x}^{*}<1 \\
& 0 \tau_{y}^{*}<1
\end{aligned}
$$

where $\tau_{\text {max }}, \tau_{x}^{*}, \tau_{y \max }$ and $\tau_{y}^{*}$ are some unknown, positive constants. Hence, the time delays are uncertain within unknown upper bounds.

\subsection{Specification}

The primary control objective is asymptotically exact tracking by the output $y(t)$ of the output $y_{r}(t)$ of a stable reference model

$$
\dot{x}_{r}(t)=A_{r} x_{r}(t)+b_{r}\left(r_{n}(t)+u_{c}(t)\right) \quad y_{r}(t)=c_{r}^{T} x_{r}(t),
$$

where $x_{r} \in \mathbb{R}^{n}, r_{n}(t), u_{c}(t), y_{r}(t) \in \mathbb{R}$, i.e. $\lim _{t \rightarrow \infty} e(t)=0$, where $e(t)=y(t)-y_{r}(t)$. In (3) the "fixed" part $r_{n}(t)$ of the reference input is in the form of a standard reference signal of adaptive control theory, Ioannou and Sun (1996), and the tolerence component of the command signal $u_{c}(t)$ may vary within specified given limits

$$
u_{c}(t) \in\left[u_{c}^{-}(t) u_{c}^{+}(t)\right] .
$$

which determine the performance tube of the reference model (3).

The transfer function of the reference model is expressed as

$$
W_{r}(s)=c_{r}^{T}\left(s I_{n}-A_{r}\right)^{-1} b_{r}=k_{r} \frac{N_{r}(s)}{D_{r}(s)}
$$

where $N_{r}(s), D_{r}(s)$ are monic polynomials and $k_{r}$ is a constant.

The second control objective is formalized as the following optimization task:

$$
\begin{aligned}
\operatorname{minimize}\left(\text { w.r.t. } u_{c}\right) & J\left(u_{c}\right)=u^{2}(t) \\
\text { subject to } & u_{c}^{-}(t) \leq u_{c}(t) \leq u_{c}^{+}(t) .
\end{aligned}
$$

\subsection{Main assumptions}

The following assumptions are made on the plant (1) and the reference model (3): (A1) When there are no terms with time delays $\tau_{x}, \tau_{y}$ and external disturbance, the plant (1) can be described by

$$
y=W_{0}(s) u, \quad W_{0}(s)=c^{T}(I s-A)^{-1} b=k_{p} \frac{N(s)}{D(s)}
$$

where $W_{0}(s)$ is the transfer function associated with the undelayed, undisturbed plant; $D(s)$ is a monic polynomial of degree $n$; the polynomial $N(s)$ is monic and Hurwitz and of degree $n-1$, i.e. the plant is minimum phase; the high-frequency plant gain $k_{p}$ is constant with known sign; (A2) The transfer function $W_{r}(s)$ is strictly positive real (SPR); (A3) $|d(t)| \leq d^{*}$, where $d^{*}$ is unknown and (A4) $A_{\tau}=b a_{\tau}^{* T}$. These assumptions are the same as in Mirkin and Gutman (2010b).

In addition to (A1)-(A4) we include the following assumption for the nonlinear function $f\left(y(t), y\left(t-\tau_{y}(t)\right), t\right)$. (A5) The function $f\left(y(t), y\left(t-\tau_{y}(t)\right), t\right)$ is assumed to be bounded by

$$
\begin{aligned}
f\left(y(t), y\left(t-\tau_{y}(t)\right), t\right) \leq & \sum_{j=1}^{p_{1}} \xi_{1 j}^{*}\left|y(t) \phi_{j}(y(t))\right| \\
& +\sum_{j=1}^{p_{2}} \xi_{2 j}^{*} \mid y\left(t-\tau_{y}(t)\right) \varphi_{j}\left(y\left(t-\tau_{y}(t)\right) \mid\right.
\end{aligned}
$$

where $p_{1}$ and $p_{2}$ are known; $\phi_{l}(\star)$ and $\varphi_{j}(\star),(j=1, \ldots, p)$ are known bounded nonlinear functions and $\xi_{1 j}^{*}$ and $\xi_{2 j}^{*}$ are unknown positive constants.

Remark 1. The minimum phase assumption is fundamental in MRAC schemes, see Ioannou and Sun (1996); Tao (2003). The assumption that the relative degree be one focusses on the simplest case amenable to Lyapunov designs; however, the paper's main idea of the new control parametrization can be extended to higher relative degree, for which case it is required to use "error augmentation" and/or "tuning error normalization", see Ioannou and Sun (1996).

\section{CONTROLLER DESIGN}

We are looking for a control strategy in the form

$$
u(t)=u_{a}(t)+u_{c}(t)
$$

where $u_{a}(t)$ is an adaptive control part and $u_{c}(t)$ is the tolerance component. Following the two phase design procedure, see 
Section 2, the two types of control laws $u_{a}(t)$ and $u_{c}(t)$ will be derived separately. In the adaptive control design phase, we are looking for an adaptive control component parametrization to guarantee the asymptotic error $e(t)$ to be equal to zero for any the command signal of the reference model (3). In the tolerance control design phase, we derive the tolerance control, $u_{c}(t)$ in order to to minimize the performance index (6). So, the adaptive control strategy modifies the controller parameters whereas the tolerance control component modifies the reference model input.

\subsection{Adaptive part parametrization}

We propose the following structure of $u_{a}(t)$ :

$$
\begin{aligned}
u_{a}(t) & =\theta^{T}(t) \omega(t)+\theta_{I}(t)+u_{n c}(t) \\
u_{n c}(t) & =-\sum_{j=0}^{p_{1}} k_{1 j} e(t) \phi_{j}^{2}(e(t))-\sum_{j=0}^{p_{2}} k_{2 j} e(t) \varphi_{j}^{2}(e(t)) \\
\dot{\theta}(t) & =-\operatorname{sgn}\left(\rho^{*}\right) \Gamma \omega(t) e(t) \\
\dot{\theta}_{I}(t) & =-\operatorname{sgn}\left(\rho^{*}\right) \gamma_{I} e(t)
\end{aligned}
$$

where $\theta(t)=\left[\theta_{e}(t) \theta_{1}^{T}(t) \theta_{2}^{T}(t)\right]^{T} \in \mathbb{R}^{2 n-1}$ and $\theta_{I} \in \mathbb{R}^{1}$ are the matrix and scalar adaptation gains respectively; the signal vector $\omega(t)=\left[e(t) x_{1}^{T}(t) x_{2}^{T}(t)\right]^{T} \in \mathbb{R}^{2 n-1}$ and $x_{1} \in \mathbb{R}^{n-1}$ and $x_{2} \in \mathbb{R}^{n-1}$ are the states of the auxiliary filters

$$
\begin{aligned}
& \dot{x}_{1}(t)=A_{f} x_{1}(t)+b_{f} u(t), x_{1}(0)=0 \\
& \dot{x}_{2}(t)=A_{f} x_{2}(t)+b_{f} y(t), x_{2}(0)=0
\end{aligned}
$$

where $A_{f} \in \mathbb{R}^{(n-1) \times(n-1)}$ is Hurwitz and $b_{f}$ is a constant vector such that $\left(A_{f}, b_{f}\right)$ is controllable. The positive scalars $k_{1 j}>0$, $k_{2 j}>0, j=1, \ldots, p, \gamma_{I}>0$ and the matrix $\Gamma=\Gamma^{T}>0$ are design parameters.

The control components $\theta^{T}(t) \omega(t), \theta_{I}(t)$ and $u_{n c}(t)$ are used simultaneously to maintain robust tracking performance with $e(t) \rightarrow 0$ in face of parametric uncertainties, bounded external disturbances and nonlinear delayed perturbations, respectively.

Remark 2. The tracking objective is achieved by output feedback only. As in Mirkin and Gutman (2010b), but in contrast to standard MRAC schemes, the feedback signal $\omega(t)$ is used in the tracking error instead of the plant output. Moreover, unlike the standard schemes, we remove the adjustable feedforward term driven by the reference model input. However as an extension to Mirkin and Gutman (2010b), we consider a rather general class of plants and therefore, to handle the plant non-linearities, a new feedback compensator term $u_{n c}(t)$ is incorporated, with adaptive error feedback. Hence the adaptive controller parametrization is a new, alsoo in the case when $u_{c}(t)=0$, i.e. in the case we do have the additional optimization problem.

Remark 3. For adaptation a proportional-integral-time delay (PITD) type algorithm can be chosen which may have better transient adaptation performance than the traditional I and PI schemes, see e.g. Mirkin and Gutman (2010b).

\subsection{Error Equation}

By using the conventional technique of model reference adaptive control Ioannou and Sun (1996); Tao (2003) the tracking error $e(t)=y(t)-y_{r}(t)$ for any $u(t)$ can be expressed as

$$
\begin{aligned}
e= & W_{r}(s) \rho^{*}\left[u-\theta_{e}^{*} y-\theta_{1}^{* T} x_{1}-\theta_{2}^{* T} x_{2}-\theta_{r}^{*}\left(r_{n}(t)+u_{c}(t)\right)\right. \\
& +H(s) d(t)+H(s) f\left(y, y\left(t-\tau_{y}(t)\right), t\right)+a_{\tau}^{* T} x\left(t-\tau_{x}(t)\right) \\
& \left.-\theta_{1}^{* T} H_{f}(s) a_{\tau}^{* T} x\left(t-\tau_{x}(t)\right)\right]
\end{aligned}
$$

where $\rho^{*}=\theta_{r}^{*-1}=k_{p} k_{r}^{-1}, \theta_{1}^{*}, \theta_{2}^{*} \in \mathbb{R}^{n-1}, \quad \theta_{e}^{*} \in \mathbb{R}, \quad \theta_{r}^{*} \in \mathbb{R}$ are so-called matching parameters, $H(s)=1-\theta_{1}^{* T} H_{f}(s)$ with $H_{f}(s)=\left(s I-A_{f}\right)^{-1} b_{f}=\frac{\left[s^{n-2} \ldots s 1\right]^{T}}{\lambda(s)} \in \mathbb{R}^{n-1}, \lambda(s)=s^{n-1}+$ $\cdots+\lambda_{1} s+\lambda_{0}$ is a monic Hurwitz polynomial and $A_{f}, b_{f}$ are from (12).

To find a suitable error equation parametrization, we manipulate the last term of (13). Firstly, we introduce a new dynamical system

$$
z(t)=\theta_{1}^{* T} H_{f}(s)\left[a_{\tau}^{* T} x\left(t-\tau_{x}(t)\right)\right]=\theta_{z}^{* T} z_{x}(t)
$$

where $\theta_{z}^{* T}=\left[\theta_{11}^{*} a_{\tau}^{* T}, \theta_{12}^{*} a_{\tau}^{* T}, \ldots, \theta_{1(n-1)}^{*} a_{\tau}^{* T}\right]$ and

$$
\begin{gathered}
z_{x}(t)=H_{n}(s)\left[x\left(t-\tau_{x}(t)\right)\right] \\
H_{n}(s)=\frac{\left[I_{n \times n} s^{n-2}, \ldots, I_{n \times n} s, I_{n \times n}\right]^{T}}{\lambda(s)}
\end{gathered}
$$

Here $\theta_{z}^{*} \in \mathbb{R}^{n(n-1)}, z_{x} \in \mathbb{R}^{n(n-1)}, H_{n}(s) \in \mathbb{R}^{n(n-1) \times n}$ and $I_{n \times n}$ is the $n \times n$ identity matrix.

Secondly we decompose the signals $z_{x j}(t)$ in (15) into two components $z_{x}(t)=z_{e}(t)+z_{r}(t)$ where

$$
\begin{aligned}
z_{e}(t) & =H_{n}(s)\left[e_{x}\left(t-\tau_{x}(t)\right)\right] z_{r}(t)=H_{n}(s)\left[x_{r}\left(t-\tau_{x}(t)\right)\right] \\
e_{x}\left(t-\tau_{x}(t)\right) & =x\left(t-\tau_{x}(t)\right)-x_{r}\left(t-\tau_{x}(t)\right)
\end{aligned}
$$

where $x_{r}(t) \in \mathbb{R}^{n}$ is the state of the reference model (3) with the state space triple $\left(A_{r}, b_{r}, c_{r}\right)$.

Then, using (14) and (17), we obtain from (13) the basic error equation

$$
\begin{aligned}
e= & W_{r}(s) \rho^{*}\left[u-\theta_{e}^{*} e(t)-\theta_{1}^{* T} x_{1}(t)-\theta_{2}^{* T} x_{2}(t)-\theta_{r}^{*}\left(r_{n}+u_{c}\right)\right. \\
& -\theta_{e}^{*} y_{r}(t)+a_{\tau}^{* T} x_{r}\left(t-\tau_{x}(t)\right)-\theta_{z}^{* T} z_{r}(t)+a_{\tau}^{* T} e_{x}\left(t-\tau_{x}(t)\right) \\
& \left.-\theta_{z}^{* T} z_{e}(t)+H(s)[d(t)]+H(s)\left[f\left(y, y\left(t-\tau_{y}(t)\right), t\right)\right]\right]
\end{aligned}
$$

Remark 4. Note that $e_{x}\left(t-\tau_{x}(t)\right)$ and $z_{e}(t)$ are not available for measurement, and we shall use them only for analysis, and not for the implementation.

Introducing the parameter vector $\theta^{*}=\left[\begin{array}{lll}\theta_{e}^{*} & \theta_{1}^{* T} & \theta_{2}^{* T}\end{array}\right]^{T} \in \mathbb{R}^{2 n-1}$ the basic tracking error equation (18) can be expressed as

$$
\begin{aligned}
e= & W_{r}(s) \rho^{*}\left[u(t)-\theta^{* T} \omega(t)-\theta_{r}^{*}\left(r_{n}(t)+u_{c}(t)\right)-\theta_{e}^{*} y_{r}(t)\right. \\
& -\theta_{z}^{* T} z_{e}(t)+a_{\tau}^{* T} x_{r}\left(t-\tau_{x}(t)\right)-\theta_{z}^{* T} z_{r}(t)+a_{\tau}^{* T} e_{x}\left(t-\tau_{x}(t)\right) \\
& \left.+H(s)[d(t)]+H(s)\left[f\left(y, y\left(t-\tau_{y}(t)\right), t\right)\right]\right]
\end{aligned}
$$

\subsection{Stability Proof}

To prove stability, the usual way of MRAC for delay free systems is used, see e.g. Ioannou and Sun (1996). The augmented vector $\hat{x}(t)=\left[\begin{array}{lll}x & x_{1} & x_{2}\end{array}\right]^{T}$ is introduced, and the state of the corresponding non-minimal realization $\hat{c}^{T}(s I-\hat{A})^{-1} \hat{b} \theta_{r}^{*}$ of $W_{r}$ is denoted by $\hat{x}_{r}(t)$. Then we can write the following state space representation for (19) 


$$
\begin{aligned}
\frac{d \hat{e}(t)}{d t}= & \hat{A} \hat{e}(t)+\bar{b} \rho^{*}\left\{u(t)-\theta^{* T} \omega(t)+a_{\tau}^{* T} \hat{l}^{T} \hat{e}\left(t-\tau_{x}(t)\right)\right. \\
& -\theta_{z}^{* T} C_{e} \hat{z}_{e}(t)-\theta_{r}^{*}\left(r_{n}(t)+u_{c}(t)\right)-\theta_{e}^{*} y_{r}(t) \\
& +a_{\tau}^{* T} x_{r}\left(t-\tau_{x}(t)\right)-\theta_{z}^{* T} H_{n}(s)\left[x_{r}\left(t-\tau_{x}(t)\right)\right] \\
& \left.+H(s)[d(t)]+H(s)\left[f\left(y, y\left(t-\tau_{y}(t)\right), t\right)\right]\right\} \\
\frac{d \hat{z}_{e}(t)}{d t}= & A_{e} \hat{z}_{e}(t)+B_{e} \hat{l}^{T} \hat{e}\left(t-\tau_{x}(t)\right), z_{e}(t)=C_{e} \hat{z}_{e}(t) \\
e(t)= & y(t)-y_{r}(t)=\hat{c}^{T} \hat{e}(t), \hat{l}=\left[I_{n \times n} 0_{n \times(n-1)} 0_{n \times(n-1)}\right]^{T}
\end{aligned}
$$

where the triple $\left(A_{e}, B_{e}, C_{e}\right)$ is a minimal state space realization of the stable transfer matrix $H_{n}(s)$ in (17), $\bar{b}=\hat{b} \theta_{r}^{*}$, and $0_{n \times(n-1)}$ is the zero $n \times(n-1)$ matrix.

Because $\hat{c}^{T}(s I-\hat{A})^{-1} \hat{b} \theta_{r}^{*}=W_{r}$ is SPR, there exists a matrix $P=P^{T}>0$ satisfying

$$
\hat{A}^{T} P+P \hat{A}+\zeta \zeta^{T}+v Q=0, \quad P \hat{b} \theta_{r}^{*}=P \bar{b}=\hat{c},
$$

where $\zeta$ is a vector, $Q=Q^{T}>0$ is any positive definite matrix and $v>0$ is some selective constant, all of which is implied by the Meyer-Kalman-Yakubovich lemma (Ioannou and Sun, 1996, pp.129-130). Since $A_{e}$ in (20) is stable, it also holds that

$$
A_{e}^{T} P_{z}+P_{z} A_{e}+Q_{z}=0
$$

where $P_{z}=P_{z}^{T}>0$ and $Q_{z}=Q_{z}^{T}>0$.

For the stability analysis we use the following LyapunovKrasovskii type functional

$$
\begin{aligned}
& \begin{aligned}
V & =\sum_{i}^{4} V_{i}, V_{1}=\hat{e}^{T} P \hat{e}+\hat{z}_{e}^{T} P_{z} \hat{z}_{e} \\
V_{2} & =\frac{v}{2} \int_{t-\tau_{x}(t)}^{t} \hat{e}^{T}(s) Q \hat{e}(s) d s+\sum_{j=1}^{p_{2}} \varsigma_{j} \int_{t-\tau_{y}(t)}^{t} e^{2}(s) \varphi_{j}^{2}(e(s)) d s \\
V_{3} & =\left|\rho^{*}\right| \tilde{\chi}^{T}(t) \Gamma^{-1} \tilde{\chi}(t) \\
V_{4} & =\left|\rho^{*}\right| \gamma_{I}^{-1}\left(\theta_{I}(t)+\theta_{I}^{*} \operatorname{sgn}(e(t))\right)^{2}
\end{aligned} \\
& \text { where } \Gamma>0 \text { is from }(11) \text { and } \\
& \qquad \tilde{\chi}(t)=\tilde{\theta}(t)+\theta_{0}
\end{aligned}
$$

The signum function $\operatorname{sgn}(e(t)$ is defined such that $\operatorname{sgn}(e(t))=$ 1 , if $e(t)>0 ; \operatorname{sgn}(e(t))=0$, if $e(t)=0$; and $\operatorname{sgn}(e(t))=$ -1 , if $e(t)<0$.

The vector

$$
\theta_{0}=\left[\frac{r_{0}}{2 \rho^{*}}, 0, \ldots, 0\right]^{T}
$$

has the same dimension as $\theta$. The unknown vector $\theta_{I}^{*}$ and the selective parameters $r_{0}>0, \varsigma_{j}>0$ will be defined later.

Using (21) and (22), the time derivatives $\dot{V}_{i}(t), i=1, \ldots, 4$ of (23) along (20) can be written

$$
\begin{aligned}
\left.\dot{V}_{1}(t)\right|_{(20)}= & -v \hat{e}^{T}(t) Q \hat{e}(t)-\hat{e}^{T}(t) \zeta \zeta^{T} \hat{e}(t)-\hat{z}_{e}^{T}(t) Q_{z} \hat{z}_{e}(t) \\
& +2 \hat{e}^{T}(t) P \bar{b} \rho^{*} u_{a}(t)+2 \hat{e}^{T}(t) P \bar{b} \rho^{*} a_{\tau}^{* T} \hat{l}^{T} \hat{e}\left(t-\tau_{x}(t)\right) \\
& -2 \rho^{*} \hat{e}^{T}(t) P \bar{b} \theta^{* T} \omega(t)-2 \hat{e}^{T}(t) P \bar{b} \rho^{*} \theta_{z}^{* T} C_{e} \hat{z}_{e}(t) \\
& +2 \hat{z}_{e}^{T}(t) P_{z} B_{e} \hat{l}^{T} \hat{e}\left(t-\tau_{x}(t)\right) \\
& -2 \hat{e}^{T}(t) P \bar{b} \rho^{*} \theta_{e}^{*} y_{r}(t)+2 \hat{e}^{T}(t) P \bar{b} \rho^{*} H(s)[d(t)] \\
& -2 \hat{e}^{T}(t) P \bar{b} \rho^{*} \theta_{z}^{* T} H_{n}(s)\left[x_{r}\left(t-\tau_{x}(t)\right)\right] \\
& +2 \hat{e}^{T}(t) P \bar{b} \rho^{*} a_{\tau}^{* T} x_{r}\left(t-\tau_{x}(t)\right) \\
& +2 \hat{e}^{T}(t) P \bar{b} \rho^{*} H(s)\left[f\left(y, y\left(t-\tau_{y}(t)\right), t\right)\right] \\
& -2 \hat{e}^{T}(t) P \bar{b} \rho^{*}\left[\theta_{r}^{*} r(t)+\theta_{r}^{*} u_{c}(t)-u_{c}(t)\right]
\end{aligned}
$$

In view the known fact that for any vectors $x, y$, and any positive-definite matrix $S$ of appropriate dimensions, it holds that $2 x^{T} y \leq x^{T} S x+y^{T} S^{-1} y$ by which we can estimate some of the terms in (26) as follows

$$
\begin{aligned}
2 \hat{e}^{T}(t) P \bar{b} \rho^{*} a_{\tau}^{* T} \hat{l}^{T} \hat{e}\left(t-\tau_{x}(t)\right) \leq & \hat{e}^{T}(t) P \bar{b} \Psi_{1} \bar{b}^{T} P \hat{e}^{T}(t) \\
& +\hat{e}^{T}\left(t-\tau_{x}(t)\right) S \hat{e}\left(t-\tau_{x}(t)\right) \\
-2 \hat{e}^{T}(t) P \bar{b} \rho^{*} \theta_{z}^{* T} C_{e} \hat{z}_{e}(t) \leq & \hat{e}^{T}(t) P \bar{b} \Psi_{2} \bar{b}^{T} P \hat{e}(t) \\
& +\hat{z}_{e}^{T}(t) S \hat{z}_{e}(t) \\
2 \hat{z}_{e}^{T}(t) P_{z} B_{e} \hat{l}^{T} \hat{e}\left(t-\tau_{x}(t)\right) \leq & \hat{e}^{T}\left(t-\tau_{x}(t)\right) \Psi_{3} \hat{e}\left(t-\tau_{x}(t)\right) \\
& +\hat{z}_{e}^{T}(t) S \hat{z}_{e}(t)
\end{aligned}
$$

where

$$
\begin{aligned}
& \Psi_{1}=\rho^{* 2} a_{\tau}^{* T} \hat{l}^{T} S^{-1} \hat{l}_{\tau}^{*}, \quad \Psi_{2}=\rho^{* 2} \theta_{z}^{* T} C_{e} S^{-1} C_{e}^{T} \theta_{z}^{*}, \\
& \Psi_{3}=\hat{l} B_{e}^{T} P_{z}^{T} S^{-1} P_{z} B_{e} \hat{l}^{T}
\end{aligned}
$$

Using boundedness of the reference signals, the correction control input $u_{c}(t)$ and the external disturbance $d(t)\left(\left|r_{n}(t)\right| \leq\right.$ $r^{*},\left|y_{r}(t)\right| \leq y_{r}^{*},\left|u_{c}(t)\right| \leq \delta=\frac{1}{2}\left[\left|u_{c}^{+}+u_{c}^{-}\right|+\left|u_{c}^{+}-u_{c}^{-}\right|\right]$and $\left.|d(t)| \leq d^{*}\right)$, the stability of the transfer functions $H_{f}(s)$ and $H(s)$ and in view of (8) we can write the following estimates for the other terms of (26):

$$
\begin{aligned}
\Xi_{e q}= & -2 \hat{e}^{T}(t) P \bar{b} \rho^{*}\left[\theta_{r}^{*} r(t)+\theta_{r}^{*} u_{c}(t)-u_{c}(t)\right] \\
& -2 \hat{e}^{T}(t) P \bar{b} \rho^{*} \theta_{e}^{*} y_{r}(t) \\
& +2 \hat{e}^{T}(t) P \bar{b} \rho^{*} a_{\tau}^{* T} x_{r}\left(t-\tau_{x}(t)\right)+2 \hat{e}^{T}(t) P \bar{b} \rho^{*} H(s)[d(t)] \\
& -2 \hat{e}^{T}(t) P \bar{b} \rho^{*} \theta_{z}^{* T} H_{n}(s)\left[x_{r}\left(t-\tau_{x}(t)\right)\right] \\
\leq & 2|e(t)|\left|\rho^{*}\right|\left(\left|\theta_{r}^{*}\right| r^{*}+\left|\theta_{r}^{*}\right| \delta+\delta+\left|\theta_{e}^{*}\right| y_{r}^{*}+\left\|a_{\tau}^{*}\right\| x_{r}^{*}\right. \\
& \left.+\left\|\theta_{z}^{*}\right\|\left\|H_{n}(s)\right\|_{\infty} x_{r}^{*}+\|H(s)\|_{\infty} d^{*}\right) \\
\leq & 2|e(t)|\left|\rho^{*}\right| \eta_{1}
\end{aligned}
$$

where

$$
\begin{aligned}
\eta_{1}= & \left|\theta_{r}^{*}\right| r^{*}+\left|\theta_{r}^{*}\right| \delta+\delta+\left|\theta_{e}^{*}\right| y_{r}^{*}+\left\|a_{\tau}^{*}\right\| x_{r}^{*} \\
& +\left\|\theta_{z}^{*}\right\|\left\|H_{n}(s)\right\|_{\infty} x_{r}^{*}+\|H(s)\|_{\infty} d^{*}
\end{aligned}
$$

For the term $\Xi_{f}=2 \hat{e}^{T}(t) P \bar{b} \rho^{*} H(s)[f(\star)]$ we have

$$
\begin{aligned}
\Xi_{f} \leq & 2|e(t)|\left|\rho^{*}\right| \mid H(s) \|_{\infty}\left(\sum_{j=1}^{p_{1}} \xi_{1 j}^{*}|e(t)|\left|\phi_{j}(e(t))\right|\right. \\
& +\sum_{j=1}^{p_{2}} \xi_{2 j}^{*}\left|e\left(t-\tau_{y}(t)\right)\right| \mid \varphi_{j}\left(e\left(t-\tau_{y}(t)\right) \mid\right. \\
& +\sum_{j=1}^{p_{1}} \xi_{1 j}^{*}\left|y_{r}(t)\right|\left|\phi_{j}\left(y_{r}(t)\right)\right| \\
& +\sum_{j=1}^{p_{2}} \xi_{2 j}^{*}\left|y_{r}\left(t-\tau_{y}(t)\right)\right| \mid \varphi_{j}\left(y_{r}\left(t-\tau_{y}(t)\right) \mid\right) \\
\leq & 2|e(t)|\left|\rho^{*}\right||| H(s) \|_{\infty}\left(\sum_{j=1}^{p_{1}} \xi_{1 j}^{*}|e(t)|\left|\phi_{j}(e(t))\right|\right. \\
& +\sum_{j=1}^{p_{2}} \xi_{2 j}^{*}\left|e\left(t-\tau_{y}(t)\right)\right| \mid \varphi_{j}\left(e\left(t-\tau_{y}(t)\right) \mid+\eta_{2}\right)
\end{aligned}
$$

where the unknown constant

$$
\eta_{2}=\sum_{j=1}^{p_{1}} \xi_{1 j}^{*} y_{r}^{*} \phi_{j}^{*}+\sum_{j=1}^{p_{2}} \xi_{2 j}^{*} y_{r}^{*} \varphi_{j}^{*}
$$

Using (2) for the time derivative $\dot{V}_{2}(t)$ of (23) we obtain 


$$
\begin{aligned}
\left.\dot{V}_{2}(t)\right|_{(20)} \leq & \frac{v}{2} \hat{e}^{T}(t) Q \hat{e}(t)-\frac{\bar{v}}{2} \hat{e}^{T}\left(t-\tau_{x}(t)\right) Q \hat{e}\left(t-\tau_{x}(t)\right) \\
& -\sum_{j=1}^{p_{2}} 2 \varsigma_{j}^{*} e^{2}\left(t-\tau_{y}(t)\right) \varphi_{j}^{2}\left(e\left(t-\tau_{y}(t)\right)\right) \\
& +\sum_{j=1}^{p_{2}} \varsigma_{j}^{*} e^{2}(t) \varphi_{j}^{2}(e(t))
\end{aligned}
$$

where $\bar{v}=\frac{v \bar{\tau}_{x}}{2}, \bar{\tau}_{x}=1-\tau_{x}^{*}>0$ and $\varsigma_{j}^{*}=\left(1-\tau_{y}^{*}\right) \varsigma_{j}$.

For the derivative $\dot{V}_{3}(t)$ in view of (25) we have

$$
\begin{aligned}
\left.\dot{V}_{3}(t)\right|_{(20)} & =-2 \rho^{*} \tilde{\theta}^{T}(t) \omega(t) e(t)-r_{0} \hat{e}^{T}(t) P \bar{b} \bar{b}^{T} P \hat{e}(t) \\
& =-2 \rho^{*} \tilde{\theta}^{T}(t) \omega(t) e(t)-r_{0} e^{2}(t)
\end{aligned}
$$

Here, we used the fact that $e(t)=\hat{c}^{T} \hat{e}(t)$, see (20).

For convenience, let $Q$ from (21), $Q_{z}$ from (22) and $r_{0}$ from (25) be $Q=Q_{1}+Q_{2}, Q_{z}=Q_{z 1}+Q_{z 2}, r_{0}=r_{01}+r_{02}+r_{03}$ with $Q_{1}=Q_{1}^{T}>0, Q_{2}=Q_{2}^{T}>0, Q_{z 1}=Q_{z 1}^{T}>0, Q_{z 2}=Q_{z 2}^{T}>0$ and $r_{01}>0, r_{02}>0, r_{03}>0$. Then, combining $-r_{01} e^{2}(t)$ and $-r_{02} e^{2}(t)$ from (32) and the first two terms from (30), completing the squares, and dropping negative terms, we obtain

$$
\begin{array}{r}
\sum_{j=1}^{p_{1}}\left(-r_{01} \frac{1}{p_{1}} e^{2}(t)+2|e(t)|\left|\rho^{*}\right|\|H(s)\|_{\infty} \xi_{1 j}^{*}|e(t)|\left|\phi_{j}(e(t))\right|\right) \\
\leq \sum_{j=1}^{p_{1}} \frac{1}{r_{01}} \zeta_{1 j} e^{2}(t) \phi_{j}^{2}(e(t))
\end{array}
$$

$$
\begin{aligned}
& \sum_{j=1}^{p_{2}}\left(-r_{02} \frac{1}{p_{2}} e^{2}(t)\right. \\
& +2|e(t)|\left|\rho^{*}\right|\|H(s)\|_{\infty} \xi_{2 j}^{*}\left|e\left(t-\tau_{y}(t)\right)\right| \mid \varphi_{j}\left(e\left(t-\tau_{y}(t)\right) \mid\right) \\
\leq & \sum_{j=1}^{p_{2}} \frac{1}{r_{02}} \zeta_{2 j} e^{2}\left(t-\tau_{y}(t)\right) \varphi_{j}^{2}\left(e\left(t-\tau_{y}(t)\right)\right)
\end{aligned}
$$

where $\zeta_{j 1}=p_{1}\left(\rho^{*}\|H(s)\|_{\infty} \xi_{1 j}^{*}\right)^{2}$ and $\zeta_{j 2}=p_{2}\left(\rho^{*}\|H(s)\|_{\infty} \xi_{2 j}^{*}\right)^{2}$ where $\zeta_{j 1}>0$ and $\zeta_{j 2}>0$ are some appropriate unknown constants.

Applying (27), (29) and (30) to (26), and using (31), (32) and (33) yields the next equation for the time derivative of $V_{\Sigma}=V_{1}+V_{2}+V_{3}$

$$
\begin{aligned}
\left.\dot{V}_{\Sigma}\right|_{(20)} \leq & -\hat{e}^{T}(t) \frac{v}{2} Q \hat{e}(t)-\hat{e}^{T}(t) \zeta \zeta^{T} \hat{e}(t)-\hat{z}_{e}^{T}(t)\left(Q_{z}-2 S\right) \hat{z}_{e}(t) \\
& -\hat{e}^{T}(t) P \bar{b}\left(r_{03} I-\Psi_{1}-\Psi_{2}\right) \bar{b}^{T} P \hat{e}(t)+2 e(t) \rho^{*} \theta_{I}(t) \\
& -\hat{e}^{T}\left(t-\tau_{x}(t)\right)\left(\bar{v} Q-S-\Psi_{3}\right) \hat{e}\left(t-\tau_{x}(t)\right) \\
& -\sum_{j=1}^{p_{1}}\left(\frac{1}{r_{01}} \zeta_{1 j}-2 \rho^{*} k_{1 j}\right) e^{2}(t) \phi_{j}^{2}(e(t)) \\
& -\sum_{j=1}^{p_{2}}\left(\frac{1}{r_{02}} \zeta_{2 j}-2 \varsigma^{*} k_{2 j}\right) e^{2}\left(t-\tau_{y}(t)\right) \varphi_{j}^{2}\left(e\left(t-\tau_{y}(t)\right)\right)
\end{aligned}
$$

Then, selecting values for $r_{01}, r_{02}, r_{03}, Q_{2}, Q_{z 2}$ and $\varsigma_{j}$ of (31) from the inequalities

$$
\begin{aligned}
& r_{03}>\lambda_{\max }\left(\Psi_{1}+\Psi_{2}\right), \lambda_{\min }\left(\bar{v} Q_{2}\right)>\lambda_{\max }\left(S+\Psi_{3}\right), \\
& \lambda_{\min }\left(Q_{z 2}\right)>\lambda_{\max }(2 S), r_{01}>\frac{\zeta_{1 j}}{2 \rho^{*} k_{1 j}}, r_{02}>\frac{\zeta_{2 j}}{2 \varsigma^{*} k_{2 j}}
\end{aligned}
$$

we obtain from (34)

$$
\begin{aligned}
\left.\dot{V}_{\Sigma}\right|_{(20) \leq} \leq & -\hat{e}^{T}(t) \frac{v}{2} Q_{1} \hat{e}(t)-\hat{e}^{T}(t) \zeta \zeta^{T} \hat{e}(t)-\hat{z}_{e}^{T}(t) Q_{z 1} \hat{z}_{e}(t) \\
& -\hat{e}^{T}\left(t-\tau_{x}(t)\right) \bar{v} Q_{1} \hat{e}\left(t-\tau_{x}(t)\right) \\
& +2 \rho^{*}|e(t)| \theta^{*}+2 \rho^{*} e(t) \theta_{I}(t)
\end{aligned}
$$

where $\theta^{*}=\left(\eta_{1}+\|H(s)\|_{\infty} \eta_{2}\right) \operatorname{sgn}\left(\rho^{*}\right)$, with $\eta_{1}$ from (29) and $\eta_{2}$ from (30).

Remark 5. We note that the coefficient matrices $Q, Q_{z}$ and $S$ and the scalar parameters $r_{01}, r_{02}, r_{03}$ and $\zeta_{j}$ are used only for analysis and do not influence the control law. Controller gains adjust automatically to counter the non-desirable effects of delayed states, parameter uncertainties, a nonlinear perturbation and an external disturbance.

Now we write the time derivative $\dot{V}_{4}(t)$ in view of (9). For the time derivative $\dot{V}_{4}$ we consider two cases: when $e(t) \neq 0$ and $e(t)=0$

$$
\begin{aligned}
\left.\dot{V}_{4}(t)\right|_{(20)} ^{e(t) \neq 0} & =2\left|\rho^{*}\right| \gamma_{I}^{-1}\left(\theta_{I}(t)+\operatorname{sgn}(e(t)) \theta_{I}^{*}\right) \dot{\theta}_{I}(t) \\
& =-2 \rho^{*}|e(t)| \theta^{*}-2 \rho^{*} e(t) \theta_{I}(t) \\
\left.\dot{V}_{4}(t)\right|_{(20)} ^{e(t)=0} & =0
\end{aligned}
$$

Using (36) and (37) we obtain for $\dot{V}$ in (23)

$$
\begin{aligned}
\left.\dot{V}\right|_{(20) \leq} \leq & -\hat{e}^{T}(t) \frac{v}{2} Q_{1} \hat{e}(t)-\hat{e}^{T}(t) \zeta \zeta^{T} \hat{e}(t)-\hat{z}_{e}^{T}(t) Q_{z 1} \hat{z}_{e}(t) \\
& -\hat{e}^{T}\left(t-\tau_{x}(t)\right) \bar{v} Q_{1} \hat{e}\left(t-\tau_{x}(t)\right)
\end{aligned}
$$

This implies, e.g. Ioannou and Sun (1996); Tao (2003) that $V$ and, therefore, $\hat{e}(t), e(t), \hat{z}_{e}(t), \theta_{I}(t), \tilde{\theta}, \theta \in L_{\infty}$. This fact is central to the remainder of the stability analysis, which follows directly using the steps in Ioannou and Sun (1996).

\subsection{Tolerance component}

Using (3), (9) and (10) it is easy to show that the optimization problem (6) has the following solution

$$
u_{c}^{\mathrm{opt}}= \begin{cases}v(t), & \text { if } v(t) \in\left[u_{c}^{-}(t) u_{c}^{+}(t)\right] ; \\ \operatorname{argmin}\left(J\left(u_{c}^{-}\right), J\left(u_{c}^{+}\right)\right), & \text {if } v(t) \notin\left[u_{c}^{-}(t) u_{c}^{+}(t)\right]\end{cases}
$$

where $v(t)=-\theta^{T}(t) \omega(t)-\theta_{I}(t)-u_{n c}(t)$.

We summarize the main result as

Theorem 1. Consider the closed-loop system defined by the plant in (1), the controller in (9), and the updating algorithm in (11) with the reference model as in (3). Then for the bounded disturbance $d(t)$ and the nonlinearity $f(\cdot)$ satisfying the inequality (8), and for any delays $\tau_{x}(t)$ and $\tau_{y}(t)$ that satisfy (2), the following properties hold: $(i)$ all signals of the closed-loop system are bounded, (ii) $\lim _{t \rightarrow \infty} e(t)=0$ and (iii) the secondary control objective (6) is achieved.

Remark 6. Theorem 1 shows that the stability of the closedloop system and the controller parameters are completely independent of the value of the plant time-delays $\tau_{x}(t)$ and $\tau_{y}(t)$. The controller is also robust to an external disturbance.

\section{SIMULATION RESULTS}

We illustrate the results by a simple unstable second order time delay system. 


$$
\begin{aligned}
{\left[\begin{array}{c}
\dot{x}_{1}(t) \\
\dot{x}_{2}(t)
\end{array}\right]=} & {\left[\begin{array}{rr}
3 & -2 \\
1 & 0
\end{array}\right]\left[\begin{array}{l}
x_{1}(t) \\
x_{2}(t)
\end{array}\right]+\left[\begin{array}{rr}
0 & 0 \\
-0.2 & -0.1
\end{array}\right]\left[\begin{array}{l}
x_{1}\left(t-\tau_{x}(t)\right) \\
x_{2}\left(t-\tau_{x}(t)\right)
\end{array}\right] } \\
& +\left[\begin{array}{l}
3 \\
0
\end{array}\right](u(t)+f(\star)+d(t)) \\
f(\star)= & 0.5 y^{4}(t)+0.8 y(t) y^{2}\left(t-\tau_{y}(t)\right)+0.9 y^{3}\left(t-\tau_{y}(t)\right) \\
y(t)= & {\left[\begin{array}{ll}
0.6 & 0.5
\end{array}\right] x(t), \quad x(0)=\left[\begin{array}{ll}
1 & 1
\end{array}\right]^{T} }
\end{aligned}
$$

All plant parameters, the nonlinearity $f(\star)$, the disturbance $d(t)=0.2 \sin (0.2 t)$, the delays $\tau_{x}(t)=4.5+3 \sin (t)$ and $\tau_{y}(t)=$ $4.5-3 \sin (t / 2)$ are unknown to the controller. The parameter values of the adaptive controller (9) in our simulation were chosen as $A_{f}=-1, b_{f}=1$ and $\Gamma=90 I$. The command signal $r_{n}(t)$ is \pm 0.5 plus a square signal with amplitude 0.1 and basic frequency $0.5 \mathrm{rad} / \mathrm{s} . A_{r}=\left[\begin{array}{rr}-4 & -1 \\ 1 & 0\end{array}\right], b_{r}=\left[\begin{array}{l}2 \\ 0\end{array}\right]$, $c_{r}=\left[\begin{array}{ll}0.5 & 0.5\end{array}\right]^{T}$. The component $u_{n c}(t)$ from (10) is $u_{n c}(t)=$ $-9 e(t)\left|e^{3}(t)\right|^{2}-9 e^{3}(t)$.

The simulation results are shown in Figures 1-2, where we show the time responses of the plant output $y(t)$ (blue lines) and the output of the reference model $y_{r}(t)$ (red lines) for two different values of the command signal $r_{n}(t): r_{n}(t)=$ $-0.5+0.1 \sin 2 t$ (Fig. 1) and $r(t)=0.5+0.1 \sin 2 t$ (Fig. 2). The variables $y_{r}^{+}(t)$ and $y_{r}^{-}(t)$ (black lines) define the admissible range for the reference trajectories. The graphs show how the adaptive controller finds an output trajectory within the tolerance envelope $y_{r}^{-} \leq y(t) \leq y_{r}^{+}$such that the criterion (6) is minimized. In both graphs we have included also the reference trajectory $y_{r n}(t)$ (green lines) for the case with $u_{c}(t)=0$ in (4).

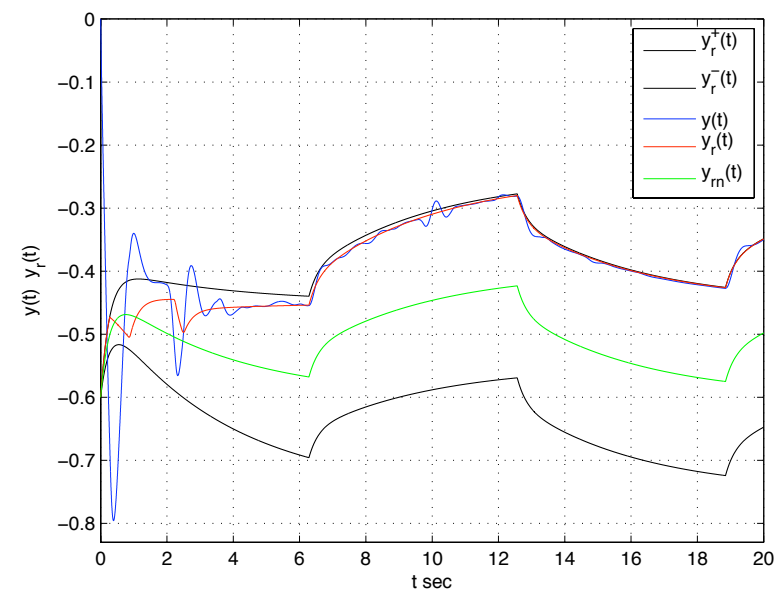

Fig. 1. Simulation of the adaptive control system for the nonlinear plant (40) and the controller (9). The graph shows how the adaptive controller finds an output trajectory within the admissible range $y_{r}^{-} \leq y_{r}(t) \leq y_{r}^{+}$.

\section{CONCLUDING REMARKS}

In this paper an initial study is presented to develop into the conventional MRAC framework an approach for solving the problem of robust adaptive output tracking for a class of uncertain nonlinear dynamical systems with unknown time-varying state delays. In common with Mirkin and Gutman (2010a), we restrict the attention to SISO systems. However, here, in contrast to Mirkin and Gutman (2010a), the presence of timevarying state delays is a distinguishing feature of the underlying

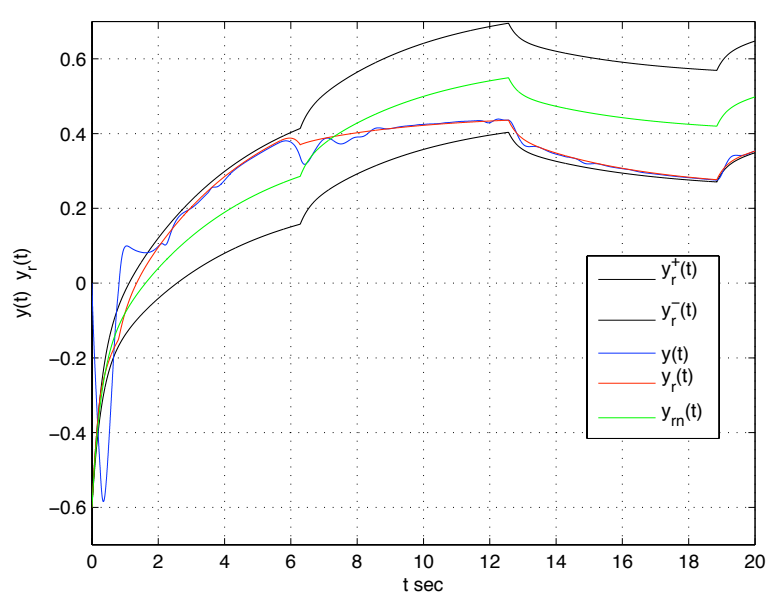

Fig. 2. Simulation of the adaptive control system for the nonlinear plant (40) and the controller (9). The graph shows how the adaptive controller finds an output trajectory within the admissible range $y_{r}^{-} \leq y_{r}(t) \leq y_{r}^{+}$.

system class. In addition to the traditional asymptotic zero error tracking specification, the MRAC problem statement includes an additional explicit requirement. The tracking objective is described by an admissible set of reference trajectories. The best trajectory is found by optimization, with a cost function that penalizes control deviations. The suggested robust adaptive controller ( $i$ ) guarantees that the tracking errors tend to zero; (ii) minimizes the control cost; and (iii) includes switching logic. To ensure the asymptotical zero tracking error, a new feedback compensator with adaptive error feedback to compensate for plant non-linearities is incorporated. The central ideas of this paper can be generalized to several other adaptive control problems.

\section{REFERENCES}

Åström, K.J. and Wittenmark, B. (1995). Adaptive control. Addison-Wesley, New York.

Ioannou, P.A. and Fidan, B. (2006). Adaptive Control Tutorial. SIAM, USA.

Ioannou, P.A. and Sun, J. (1996). Robust Adaptive Control. Prentice-Hall, New Jersey.

Krstić, M., Kanellakopoulos, I., and Kokotović, P. (1995). Nonlinear and adaptive control design. John Wiley, New York.

Mirkin, B.M. (2001). Decomposition-coordination optimization of the dynamic systems with adaptation of the criterion. Automation and Remote Control, 62(7), 1155 - 1164.

Mirkin, B.M. and Gutman, P.O. (2010a). Performance improvement in model reference adaptive control: on-line reference model adaptation. Automatica. Submitted.

Mirkin, B.M. and Gutman, P.O. (2010b). Robust adaptive output-feedback tracking for a class of nonlinear timedelayed plants. IEEE Transaction on Automatic Control, 55(10), 2418-2424.

Mirkin, B.M. and Mirkin, E.L. (1999). Adaptive decentralized control with the interval model. Vestnik MUK, 5(1), 3-10.

Narendra, K.S. and Annaswamy, A.M. (1989). Stable Adaptive Systems. Prentice-Hall, New York.

Tao, G. (2003). Adaptive Control Design and Analysis. John Wiley, New York. 